\title{
Assessment of Disease Activity by Infrared Thermography in Patients with Rheumatoid Arthritis: A Comparative Cross-Sectional Study of Thermographic, Clinical and Ultrasound Assessments
}

DOROTHEE VINSON ( $\sim$ dorothee.vinson@gmail.com )

Assistance Publique Hopitaux de Marseille https://orcid.org/0000-0002-9734-3522

Caroline paris

Assistance Publique Hopitaux de Marseille

Pierre Lafforgue

Assistance Publique Hopitaux de Marseille

Christophe Richez

Centre Hospitalier Universitaire de Bordeaux

Vincent Pradel

Assistance Publique Hopitaux de Marseille

Thao Pham

Assistance Publique Hopitaux de Marseille

Research article

Keywords: Rheumatoid Arthritis, thermal imaging, thermography, Ultrasound, clinical assessment

Posted Date: June 17th, 2020

DOl: https://doi.org/10.21203/rs.3.rs-34703/v2

License: (c) (i) This work is licensed under a Creative Commons Attribution 4.0 International License.

Read Full License 
The authors have withdrawn this preprint from Research Square 